\title{
Anwalt der Betroffenen und sozialer Unternehmensverband
}

\section{Die Position von Spitzenverbänden der Freien Wohlfahrtspflege im Spannungsfeld zwischen Politik und Geschäft}

\author{
Ulrich Schneider
}

Dr. Ulrich Schneider ist

Hauptgeschäftsführer des

Paritätischen Gesamtverbandes e. V.

Internet http://www.paritaet.org
Wohlfahrtsverbände bekommen oft die Frage gestellt, ob sie sich eher als Anwälte hilfesuchender Menschen begreifen oder als ein Unternehmensverband ihrer Mitgliedsorganisationen, die Träger von Diensten und Einrichtungen sind. Beide Funktionen gehören jedoch zusammen, da nur beide soziale Standards und soziale Angebote auf Dauern sichern können.

»Anwalt der Betroffenen « oder »sozialer Unternehmensverband « - die Alternative suggeriert mit einem forschen Entwederoder die Nichtvereinbarkeit beider Positionen oder doch zumindest eine Mühsal der Vereinbarkeit. Von einem ständigen Spannungsfeld ist dabei oft die Rede. Und in der Tat gehört es zum rhetorischen Standardprogramm von Spitzenverbandsvertretern, einen vermeintlichen Spagat zwischen diesen beiden Funktionen zu beschreiben, der doch so schmerzhaft sei, den es aber auszuhalten gelte.

Gleich anfangs meiner Ausführungen möchte ich daher aufgrund meiner verbandlichen Erfahrungen zwei Dinge richtig stellen:

- Das Spannungsverhältnis zwischen sozialer Anwaltschaft und Unternehmensverband ist kein zwangsläufiges und

- der Spagat, den es auszuhalten gilt, muss nicht gar so schmerzhaft sein.

Zweifelsfrei unterscheiden sich Spitzenverbände der Freien Wohlfahrtspflege von sogenannten »Ein-Themen-Verbänden« oder gar privat-gewerblichen Anbietern und ihren Verbandsvertretungen deutlich. Für die Wohlfahrtsverbände ist es konstitutiv, dass sie in der Tat Unternehmensverbünde darstellen und zugleich die Interessenvertretung von unterprivilegierten Gruppen der Gesellschaft oben auf ihrer Agenda haben.

Nach der aktuellen Statistik der Bundesarbeitsgemeinschaft der Freien Wohlfahrtspflege sind es 98.837 Einrichtungen und Dienste mit insgesamt 3,6
Millionen Plätzen und Betten, die die Spitzenverbände der Freien Wohlfahrtspflege vorhalten. Die Dienste werden, der Statistik der Berufsgenossenschaft folgend, durch 1,9 Millionen hauptamtlich Beschäftigte erbracht. Die Unternehmen befinden sich entweder

- in direkter Trägerschaft der Verbände

- in rechtlich selbstständigen Gesellschaften, die jedoch in der Regel von den Verbänden beherrscht werden, oder

- in einem Mitgliedschaftsverhältnis zu einem Spitzenverband der Freien Wohlfahrtspflege.

Unabhängig von der konkreten Anbindung ist den unterschiedlichen Konstellationen gemeinsam, dass in allen Fällen den Unternehmungen vor Ort eine irgendwie geartete "Zentrale «, ein organisatorisch abgetrennter Back-Office-Bereich gegenübersteht.

Eine solche Struktur hat zwangsläufig zur Folge, dass eine Interessenidentität zwischen Unternehmung und Zentrale nie vollständig bestehen kann. Wenn auch miteinander verschränkt, so bleiben es immer zwei unterschiedliche Systeme, mit unterschiedlichen Dynamiken, Alltagszielen, Organisationsinteressen - und vor allen Dingen mit unterschiedlichen Umfeldern. Aus einer solchermaßen strukturell unmöglichen Interessenidentität können sich in der Praxis unter Umständen, die meist mit dem Verbandsmanagement oder der konkreten Verfasstheit von Verbandsstrukturen selbst zu tun haben, ein atmospärisches Spannungsverhältnis und auch echte Interessengegensätze entwickeln.

Wenn somit von einem Spannungsfeld zwischen Unternehmensverbund und Sozialverband die Rede ist, so hat dies ganz wesentlich mit dieser organisatorischen Eigenart zu tun, die immer eine potenziell spannungsreiche Partnerschaft sein muss.

Wie manifestiert sich das angesprochene Spannungsfeld? Auf welchen Feldern brechen typischerweise die Probleme auf? 
Es handelt sich nach meiner Erfahrung im Wesentlichen um drei Problembereiche, in denen es zu Spannungen kommen kann: bei der Ressourcenverteilung, bei der Festlegung von Zielen und bei Fragen von Werbung und Akquise.

\section{Ressourcenverteilung}

Sozialanwaltschaft braucht Ressourcen - ebenso wie Dienstleistungen für Unternehmungen. Das Tätigkeitsspektrum eines Spitzenverbandes der Freien Wohlfahrtspflege umfasst so unterschiedliche Aufgaben wie die Sensibilisierung der Öffentlichkeit für soziale Problemlagen, den politischen Einsatz für die Interessen sozial benachteiligter Bevölkerungsgruppen oder die Auseinandersetzung mit verteilungspolitischen Fragen auf der einen Seite, aber auch Dienstleistungen für die Unternehmungen wie etwa das Einwerben von Finanzmitteln, Entgeltverhandlungen, Organisationsberatung, Vermittlung von Versicherungsschutz, Vermittlung günstiger Rahmenverträger für Betriebsmittel aller Art und vieles mehr auf der anderen Seite.

Dass bei immer nur begrenzten Ressourcen in den Zentralen die Protagonisten jeweils spezifischer Verbandsinteressen mehr Einsatz für ihre Belange fordern, liegt in der Natur der Sache. Es ist damit jedoch keinesfalls typisch oder beschränkt auf das Spannungsverhältnis von Unternehmensverbund und Sozialanwaltschaft. Die gleiche Ressourcendiskussion findet auch zwischen einzelnen Fachbereichen oder zwischen Fachbereichen und Stabsaufgaben wie Öffentlichkeitsarbeit statt. Hieraus einen Interessengegensatz zwischen Unternehmensverbund und Sozialverband abzuleiten, mag in einer aktuellen Auseinandersetzung um Ressourcen als taktisch geschickt angesehen werden, dürfte jedoch bei genauer Analyse den Kern des Problems kaum treffen.

\section{Zielkonflikte}

Nicht abgestritten werden soll, dass es bei Wohlfahrtsverbänden tatsächliche inhaltliche Zielkonflikte geben kann, die aus den beiden Funktionen Unternehmensverbund und Sozialverband erwachsen. Spätestens in der Diskussion um ge- setzliche Standards in Einrichtungen oder um Kontrollen durch die öffentliche Seite werden sie beispielhaft. Es dürfte kaum in Abrede gestellt werden, dass die Garantie bestimmter fachlicher, personeller und baulicher Standards - von Kindergärten bis zu Pflegeeinrichtungen - im Interesse der dort versorgten Personen ist. Ebenso selbstverständlich ist, dass eine Erhöhung von Standards ohne entsprechende Finanzierungsgarantien die Unternehmungen unter außerordentlichen Druck setzen und in erhebliche Schwierigkeiten bringen können.

In der Verbandspraxis entsteht daraus in der Tat ein Spannungsverhältnis spätestens dann, wenn der Verband zu konkreten politischen Vorhaben auf diesem Feld Stellung beziehen muss. Doch handelt es sich auch hierbei kaum um eine »in der Natur der Sache « liegende Gegnerschaft. Ob nämlich aus einem Spannungsverhältnis tatsächlich ein Antagonismus wird, hat tatsächlich mit den Akteuren selbst und weniger mit der Sache selbst zu tun. rechtigt. Eine vorausschauende Unternehmensstrategie wird jedoch diese Kritik immer mit einem offensiven Aufgreifen berechtigter Interessen an Standards, an Garantien und Transparenz verbinden. Wo dies geschieht und in dieser Strategie offensiv der Wettbewerbsvorteil gesucht wird, wird das Spannungsverhältnis zwischen sozialer Anwaltschaft und Unternehmensverbund nicht zum lähmenden Antagonismus, sondern zum konstruktiven und produktiven Motor gerade auch für die unternehmerische Entwicklung.

\section{Akquisestrategien}

Schließlich treten Probleme auch dann auf, wenn es um Werbung und Akquise geht. Eine konsequente sozialanwaltschaftliche Interessenvertretung verlangt gelegentlich ein etwas forscheres, eckigeres und kämpferisches Auftreten. Gleichwohl wissen wir gut, dass ein solches Gebaren wenig geeignet ist, Spender oder

\section{»Die organisatorische Trennung von Sozialverband und Unternehmensverbund ist eine Scheinlösung «}

Entscheidend ist bei einem solchen Zielkonflikt nämlich, wie Betroffeneninteressen vertreten werden, und wie Unternehmungen gemanagt und strategisch aufgestellt sind. Kaum einer käme auf die Idee, Aldi ein kluges Firmenmanagement $\mathrm{zu}$ bescheinigen, wenn sie lauthals jeglichen Verbraucherschutz verdammen würden. Dass in Asien bereits eine neue Generation energiesparender Autos vom Band läuft, während sich die deutsche Automobilindustrie im Wesentlichen durch die politische Bekämpfung von Umweltstandards einen Namen macht, wird allgemein kaum als clevere Unternehmenspolitik eingeschätzt.

Und Ähnliches gilt für Unternehmungen der Sozialwirtschaft: Die Kritik an zu vielen bürokratischen Auflagen, zu vielen Formalismen und zu großer Praxisferne derjenigen, die Richtlinien aufstellen, ist sicherlich in nicht wenigen Punkten be-
Kunden zu gewinnen. Auch wird, wer sich mit Krankenhäusern und Pflegeeinrichtungen am Markt behaupten muss, kaum aus Gründen der politischen Strategie und der Erhöhung von Durchsetzungsmacht die Situation in Pflegeeinrichtungen oder Krankenhäusern skandalieren können.

Auf dieser dritten Problemebene strategischer Unverträglichkeiten finden sich denn in der Tat die stärksten Widersprüche, die auch bei noch so klugem Verbands- und Unternehmensmanagement nicht ohne weiteres aufzulösen sind. Hier ist der eingangs zitierte Spagat gefragt, der jedoch, wird Verbandspolitik mit Augenmaß betrieben, kein so schmerzhafter sein muss, wie häufig beklagt. Vor allen Dingen sollte ein gelegentliches Ziehen und Zerren nicht zum Anlass für eine vorschnelle Flucht aus den Interessenkonflikten genommen werden. 


\section{Was tun?}

Die vermeintliche Lösung für die Probleme von Unternehmensverbund und Sozialverband ist ebenso naheliegend wie verlockend: Die Widersprüche werden aufgelöst, indem das System aufgelöst wird. Die Unternehmensteile werden in eigene organisatorische Einheiten ausgelagert, die Zuständigkeit des Verbandes und die Möglichkeiten der Einflussnahme werden strukturell auf ein Mindestmaß begrenzt, womit bei den jeweils verbleibenden Einheiten sowohl Aufgaben- wie Interessenidentitäten hergestellt wären.

Kurzfristig mag eine solche Strategie Vorteile mit sich bringen: Die eine oder andere von Widersprüchen geleitete und quälende Diskussion in Verbandsorganen wird beendet. Die unternehmerische Seite kann ohne Einmischung, die als fachfremd empfunden wird, ihre Potenziale entfalten. Entscheidungswege können effektiviert und optimiert werden. Auf der anderen Seite kann sich der Idealverein endlich wieder ganz um Ideelles kümmern. Die Last der wirtschaftlichen Verantwortung für Unternehmungen kann von den Vorstandsmitgliedern genommen werden. Die Profile und Aufgaben scheinen klarer und beherrschbarer.

Mittel- und langfristig werden jedoch die Schattenseiten einer solchen Strategie ins Auge fallen und zwar gerade für die Unternehmensseite selbst: Diese entfernt sich aus dem Zentrum des Verbandes und verliert damit ihren Einfluss auf die politische Vertretung der Organisation, die für die Unternehmen heutzutage mindestens ebenso wichtig ist wie für die betroffenen Personen. In der Folge können Interessenkonflikte zwischen Idealverband und Unternehmungen eher noch zunehmen, da der organisatorische Zwang zur Interessenverarbeitung mehr oder minder entfallen ist. Fliehkräfte sorgen für eine Einbuße an Corporate Identity, die irgendwann zwangsläufig auf die Außenwirkung durchschlagen muss: auf das Außenbild, das Image und die Marke eines Verbandes. Ein wesentlicher Wettbewerbsvorteil der den Spitzenverbänden angeschlossenen Unternehmungen - das gute Image der Wohlfahrtsverbände in der Bevölkerung - wird damit letztlich aufs Spiel gesetzt.

Nach meiner Überzeugung besteht in dem Spannungsverhältnis zwischen Unternehmensverbund und sozialer An- waltschaft der Spitzenverbände kein Wettbewerbsnachteil, sondern ein deutlicher Wettbewerbsvorteil, wenn der Konflikt klug und konstruktiv gemanagt wird. Damit ziele ich nicht nur auf die Gewinnung ehrenamtlichen Potenzials und Spenden ab. Ich meine vor allen Dingen die Verankerung der Verbände im bundesdeutschen Gemeinwesen, die jedoch nur in diesem Spannungsverhältnis denkbar ist.

\section{Resümee}

Für die Ausgliederung von Unternehmungen aus Verbandsstrukturen kann es im Einzelfall eine Vielzahl guter Gründe geben. Die organisatorische Trennung kann in der Tat Probleme lösen und Verbände weiterbringen. Für das Problem von Interessenkollisionen in der Doppelfunktion von Spitzenverbänden - Unternehmensverbund und Sozialverband stellt sie jedoch in aller Regel eine Scheinlösung dar.

Die Lösung besteht vielmehr in der steten und konstruktiven Bearbeitung des Spannungsverhältnisses selbst. Das heißt: Organisation von Kommunikation zwischen Unternehmungen und Verbandszentralen, offensives Aufgreifen der Problemanzeigen aus den Unternehmungen, eine Personalpolitik in den Verbandszentralen, die auch die Unternehmensbedürfnisse im Blick hat und ein mit Aufgeschlossenheit und Weitblick gesegnetes Management in den Unternehmungen selbst.

Der vorstehende Beitrag ist die überarbeitete Fassung eines Vortrags des Verfassers beim 5. Kongress der Sozialwirtschaft am 26. April 2007 in Magdeburg (Internet http://www.kongress-der-sozialwirtschaft.de).

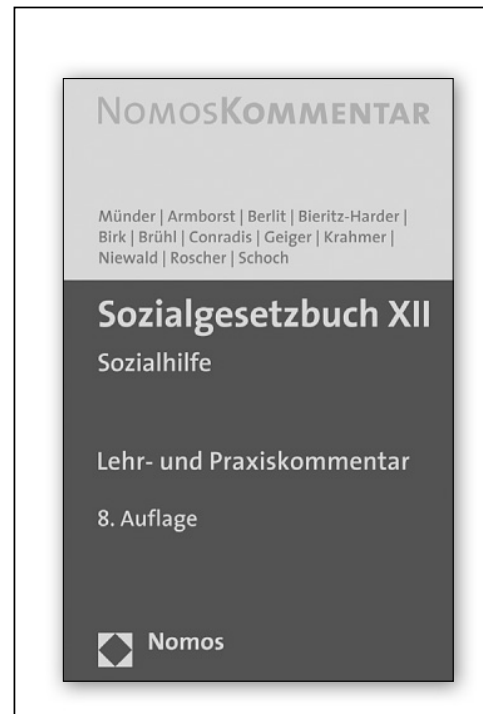

Sozialgesetzbuch XII Sozialhilfe

Lehr- und Praxiskommentar Von Prof. Dr. Johannes Münder; MinR Christian Armborst; RiBVerwG Prof. Dr. Uwe Berlit; Prof. Dr. Renate Bieritz-Harder; Prof. Dr. Ulrich-Arthur Birk; Prof. Dr. Albrecht Brühl; RA Dr. Wolfgang Conradis, FASozR; RiSG Udo Geiger; Prof. Dr. Utz Krahmer; RiLSG Stephan Niewald; Prof. Dr. Falk Roscher und RegDir a.D. Dietrich Schoch

8. Auflage 2008, 1.017 S., geb., 44,- $€$, ISBN 978-3-8329-2930-5

Die Neuauflage des LPK-SGB XII bringt Sie im Sozialhilferecht wieder auf den neuesten Stand.

"Eine unentbehrliche Arbeitshilfe. Wohl kein anderer Kommentar kann einen vergleichbaren gebündelten Sachverstand an Autoren aus Universität, Gerichten und der Anwaltschaft aufbieten." Rechtsdienst der Lebenshilfe 3/05

\section{Nomos}

